4. De Guise E, Bélanger $S$, Tinawi $S$, et al. Usefulness of the rivermead postconcussion symptoms questionnaire and the trail-making test for outcome prediction in patients with mild traumatic brain injury. Appl Neuropsychol 2016;23:213-22. doi:10.1080/23279095.2015.1038747

\section{COMPARISON OF QSOFA, AND HOSPITAL EARLY WARNING SCORES FOR PROGNOSIS IN SUSPECTED SEPSIS IN EMERGENCY DEPARTMENT PATIENTS: A SYSTEMATIC REVIEW}

${ }^{1}$ Lisa Sabir, ${ }^{2}$ Shammi Ramlakhan, ${ }^{3}$ Steve Goodacre. ${ }^{1}$ Northern General Hospital; ${ }^{2}$ Sheffield Children's Hospital NHS Foundation Trust, Western Bank, Sheffield, S10 2TH; ${ }^{3}$ School of Health and Related Research

\subsection{6/emj-2020-rcemabstracts.36}

Aims/Objectives/Background Sepsis is a major cause of morbidity and mortality and many tools exist to facilitate early recognition. The current international consensus definition of sepsis recommends the use of the quick Sequential Organ Failure Assessment (qSOFA) score in the emergency department (ED) to rapidly identify those who are likely to have poor outcomes. Early Warning Scores (EWS) are used more routinely; if these could provide the same information, they could allow standardisation and streamlining of effort.

This review compares two tools qSOFA and EWS (National/ Modified Early Warning Scores (NEWS/MEWS)) for predicting intensive care (ICU) admission and mortality when applied to suspected sepsis patients in the ED.

Methods/Design A literature search was conducted using Medline, CINAHL, Embase, and Cochrane Library, hand searching of references and a grey literature search with no language or date restrictions. Two authors selected studies and quality assessment completed using QUADAS-2. Area under the Receiver Operating Characteristic Curve (AUROC), sensitivities, and specificities were compared.

Results/Conclusions

Results 12 studies were included, totalling 395,661 patients. All reported mortality and six reported ICU admission.

AUROC estimates were variable ranging from little better than chance to good prediction. The ranges demonstrated overlap between scores suggesting little difference for predicting mortality (NEWS: 0.59-0.88; qSOFA: 0.57-0.79; MEWS 0.56-0.75). However, individual papers mostly reported higher AUROC values for NEWS than qSOFA. NEWS demonstrated a trend to better sensitivity for ICU admission (NEWS $\geq 5$ 0.46-0.91; qSOFA $\geq 2 \quad 0.12-$ 0.53 ) and mortality (NEWS $\geq 5 \quad 0.51-0.97 ; \quad$ qSOFA $\geq 2 \quad 0.14-0.7$ ) but lower specificity (ICU: NEWS $\geq 5$ 0.25-0.91; qSOFA $\geq 2$ 0.670.99 . Mortality: NEWS $\geq 5 \quad 0.22-0.91$; qSOFA $\geq 2 \quad 0.58-0.99$ ).

Conclusion The wide range of AUROC estimates and high heterogeneity limit our conclusions. Allowing for this, the NEWS AUROC was consistently higher than qSOFA within individual papers. Both scores allow threshold setting, determined by the preferred compromise between sensitivity and specificity. At established thresholds NEWS trended to higher sensitivity whilst qSOFA favoured specificity.

\section{ANALYSIS OF AN AMBULATORY CARE PATHWAY FOR PATIENTS WITH COVID-19 UTILISING REMOTE PULSE OXIMETRY}

Jonathon Kyriakides, Aria Khani, Charlotte Kelly, Reginald Coleman. Barnet Hospital, Royal Free London NHS Foundation Trust

10.1136/emj-2020-rcemabstracts.37
Aims/Objectives/Background The safe discharge of COVID-19 patients from the emergency department (ED) is difficult due to uncertainties surrounding the trajectory of the disease course. The infectivity of COVID-19 also limits followup options. A novel pathway consisting of home pulse oximetry with telephone follow-up was created to facilitate safe discharge from the ED of a London district general hospital. The primary objective was to utilise home pulse oximetry to prevent hospital admission. The secondary objective was to identify those requiring further care or investigation.

Methods/Design Adult patients with confirmed or suspected COVID-19 with oxygen saturations of between 90-94\% who were otherwise suitable for discharge were identified. These patients were discharged from the ED with a pulse oximeter. Oxygen saturations were measured three times a day for seven days. Patients received a structured telephone consultation on days two, five and seven post-discharge from the ED, and a decision was made as to whether further clinical assessment in the ED was necessary.

Results/Conclusions Of the twenty patients discharged on the pathway, $85 \%$ avoided hospital admission, whilst 15\% were re-assessed and subsequently admitted to hospital. 20\% of patients required re-assessment in the $\mathrm{ED}$ but did not require admission.

Home pulse oximetry with telephone follow-up was used to prevent hospital admission in a considerable proportion of patients who would have otherwise been admitted in the absence of this pathway. Telephone follow-up effectively identified patients who required further clinical assessment. Increasing age, the presence of co-morbidities, and pulmonary infiltrates on chest radiograph were more common in the cohort who required re-assessment. This study demonstrates the potential for safe ambulation of a subgroup of patients with COVID-19, whilst identifying practical inclusion criteria which could be replicated in ambulatory units across the UK.

\section{A CLINICAL RISK SCORE TO IDENTIFY PATIENTS WITH COVID-19 AT HIGH RISK OF CRITICAL CARE ADMISSION OR DEATH: AN OBSERVATIONAL COHORT STUDY}

James Galloway, Ruth Sneep, Fleur Cantle, Raeesa Jina, Andrew Brookes, Carole Reid, Leah Sugarman, Sam Norton. King's College Hospital

\subsection{6/emj-2020-rcemabstracts.38}

Aims/Objectives/Background The COVID-19 pandemic continues to escalate. There is urgent need to stratify patients. Understanding risk of deterioration will assist in admission and discharge decisions, and help selection for clinical studies to indicate where risk of therapy-related complications is justified.

Methods/Design An observational cohort of patients acutely admitted to two London hospitals with COVID-19 and positive SARS-CoV-2 swab results was assessed. Demographic details, clinical data, comorbidities, blood parameters and chest radiograph severity scores were collected from electronic health records. Endpoints assessed were critical care admission and death. A risk score was developed to predict outcomes. Results/Conclusions Analyses included 1,157 patients. Older age, male sex, comorbidities, respiratory rate, oxygenation, radiographic severity, higher neutrophils, higher CRP and 